\begin{tabular}{l|c|c}
\hline ISSN: 0001-5113 & ACTA ADRIAT., & SHORT COMMUNICATION \\
AADRAY & $61(2): 197-204,2020$ & \\
\hline
\end{tabular}

\title{
Length-weight and length-length relations for 21 fish species caught in Izmir Bay
}

\author{
Ali KARA ${ }^{1 *}$, Deniz ACARLI ${ }^{2}$, Akın Türker ILLKYAZ ${ }^{1}$ and Ali Özcan BABAOĞLU ${ }^{1}$ \\ ${ }^{1}$ Ege University, Faculty of Fisheries, 35100, Bornova, Izmir, Turkey \\ ${ }^{2}$ Çanakkale Onsekiz Mart University, Gökçeada School of Applied Sciences, \\ 17760, Gökçeada, Çanakkale, Turkey \\ *Corresponding author: ali.kara@ege.edu.tr
}

This study exhibits relationships between length-weight and length-length for 21 fish species caught in Izmir Bay. Coefficients $a$ and $b$ for the length-weight relationships (LWRs) and lengthlength relationships (LLRs) were calculated with $W=a L b$ formulae and as equations of $T L=a+b F L$ and $T L=a+b S L$ respectively. Equations of length-length for converting standard length and fork length into total length and vice versa were proven linear. The involved relationships were significantly interrelated (R2>0.942). Values $b$ in the LWRs varied from 2.21 to 3.96 (mean $\pm S E$ : $3.15 \pm 0.08)$. The student's t-test showed that $87.9 \%$ of values $b$ were significantly different from 3 .

Key words: Fish species, length-length relationships, length-weight relationships, Izmir Bay

\section{INTRODUCTION}

Both LWRs and LLRs are extensively employed in fisheries research and management (WANG et al., 2012; KEIVANY et al., 2015b). LWRs of fishes are widely used to weight a sample via its length and vice versa (BEYER, 1987; BINOHLAN \& PAULY, 1998; KEIVANY et al., 2015a). The LWRs study for a species can provide important insights into the biology (SARKAR et al., 2008) and the ecology (FROESE, 2006) of the species. LLRs are beneficial in a standardizing type of length when data has been outlined (FROESE, 1998) and are also functional for similar population areas (MOUTOPOULOS \& STERGIOU, 2002).

The aim of this study was to provide basic data on length-weight relationship and length- length relationship for 21 fish species sampled from Izmir Bay. The estimated LWRs and LLRs of the species could present valuable database for future researches to make comparisons between years and locations. .

\section{MATERIAL AND METHODS}

Izmir Bay $\left(38^{\circ} 32^{\prime} 09^{\prime \prime} \mathrm{N}\right.$ and $\left.26^{\circ} 45^{\prime} 18^{\prime \prime} \mathrm{E}\right)$ is one of the most significant basin concerning maritime processes and fisheries covering an area of $960 \mathrm{~km}^{2}$ with a shoreline of $464 \mathrm{~km}$ (YUCEL-GIER et al., 2010). It is a hot-spot fishing area with $83.4 \%$ of the bay allotted to fisheries (PAZI et al., 2010).

The bay is the region composed of natural reef areas, seagrass in and around Gediz flow- 
ing into the bay and related lagoons suitable for fish to spawn and feed and for some species to reproduce. It has fisheries areas and seagrass beds where fishing is conducted all year round (OZAYDIN \& TASKAVAK, 2006).

The samples of the study were collected from local commercial fishermen using gillnets, trammel nets, purse seines, longlines and hand lines and from visits to the local (i.e., Urla, Güzelbahçe and Şemikler) fishing ports between January and December 2015.

Fishes collected or bought from commercial fishing boats and auctions were made in the lab when they were freshly caught. Measurements were occasionally made by using the digital balance and measurement scale that available and ready for usage in the cooperative building at the port. Lengths (TL, SL and FL) of specimens were measured in $\mathrm{mm}$ with their weight (W) in g.

A relationship between length and weight of the species was calculated by $\mathrm{W}=a \times \mathrm{L}^{b}$ where $\mathrm{W}$ is the weight $(\mathrm{g})$ at a given $\mathrm{L}$ length $(\mathrm{cm})$, $a$ (intercept) and $b$ (slope) the regression coefficient (RICKER, 1979). Parameters of the regression coefficient were calculated through linear regression analysis on converted weight and length data and the growth type was identified by Student's t-test. Relationships of TL vs FL and TL vs SL were also computed by linear regression.

\section{RESULTS}

Analyses for regressions of LW and LL were performed on 5728 individuals from 21 species in 10 families included in the study. Table 1 presents the number of the sampling (n), minimal and maximal length-weight ranges, $\mathrm{LW}$ and $\mathrm{LL}$ relations, factors $a$ and $b$, determination $\left(\mathrm{R}^{2}\right)$.

All relations were significant $(P<0.05)$. The determination coefficient $\mathrm{R}^{2}$ was higher than 0.900 , ranging from 0.941 to 0.999 . The allometric parameter coefficient $b$ ranged from a minimum of 2.21 for Sphyraena sphyraena to a maximum of 3.96 for $T$. capelanus with a mean value of $3.15 \pm 0.08$ (mean $\pm \mathrm{SE}$ ), which was significant difference from 3 $(\mathrm{P}<0.05$, t-test $=1.86)$. Concerning kind of growth, 3, 5 and 13 species exhibited an iso- metric growth $(b=3)$ negative allometric growth $(b<3)$ and positive allometric growths $(b>3)$, respectively (Table 1).

\section{DISCUSSION}

Sampling times are different as the sampled fishes that are collected from commercial fishermen. Fishes have been collected during the months caught by commercial fishermen, that is, during the catching periods of the fish. The sample time is shown in Table 1 by numbering the months. The estimated LWR and LLR values represent the sampling time. It should not be considered as an annual average value.

The exponent values reported for " $b$ " in different fishes ranged from 2.5 to 4.0 (HILE, 1936; MARTIN, 1949). FROSE (2006) declared that $b$ prime should normally be between 2.5 and 3.5 and TESCH (1971); BAGENAL, T. \& F. W. TESCH (1978); KOUTRAKIS \& TSIKLIRAS (2003) stated that it was between 2 and 4 . The calculated allometric coefficient $b$ varied among the species from a minimum of 2.21 for S. sphyraena, to a maximum of 3.96 for T. capelanus. These values are within the limits ( 2 and 4$)$ reported by TESCH (1971) and BAGENAL \& TESCH (1978) and KOUTRAKIS \& TSIKLIRAS (2003).

Variations of values $b$ are based on the anatomic and morphological characteristics as well as on abiotic and biotic factors of their habitation when the sampling has been made (STERGIOU \& MOUTOPOULOS, 2001; MOUTOPOULOS \& STERGIOU, 2002; FROESE et al., 2011).

The factors above (abiotic and biotic factors) were out of the scope of the present study. Their effects have not been studied. It was only reported which types of different fishing gears were used and in which months the fishes were caught, thus providing the opportunity to make comparisons for further studies in the same study area (in Izmir Bay).

The $b$ value of $S$. sphyraena specimens, which were collected from the bay between September and October, was found as 2.21. PETRAKIS \& STERGIOU (1995) determined their $b$ value as 2.32 in the South Euboikos Gulf - which is almost in the same latitude and climate as Izmir Bay-with similar $b$ values at the same period of 


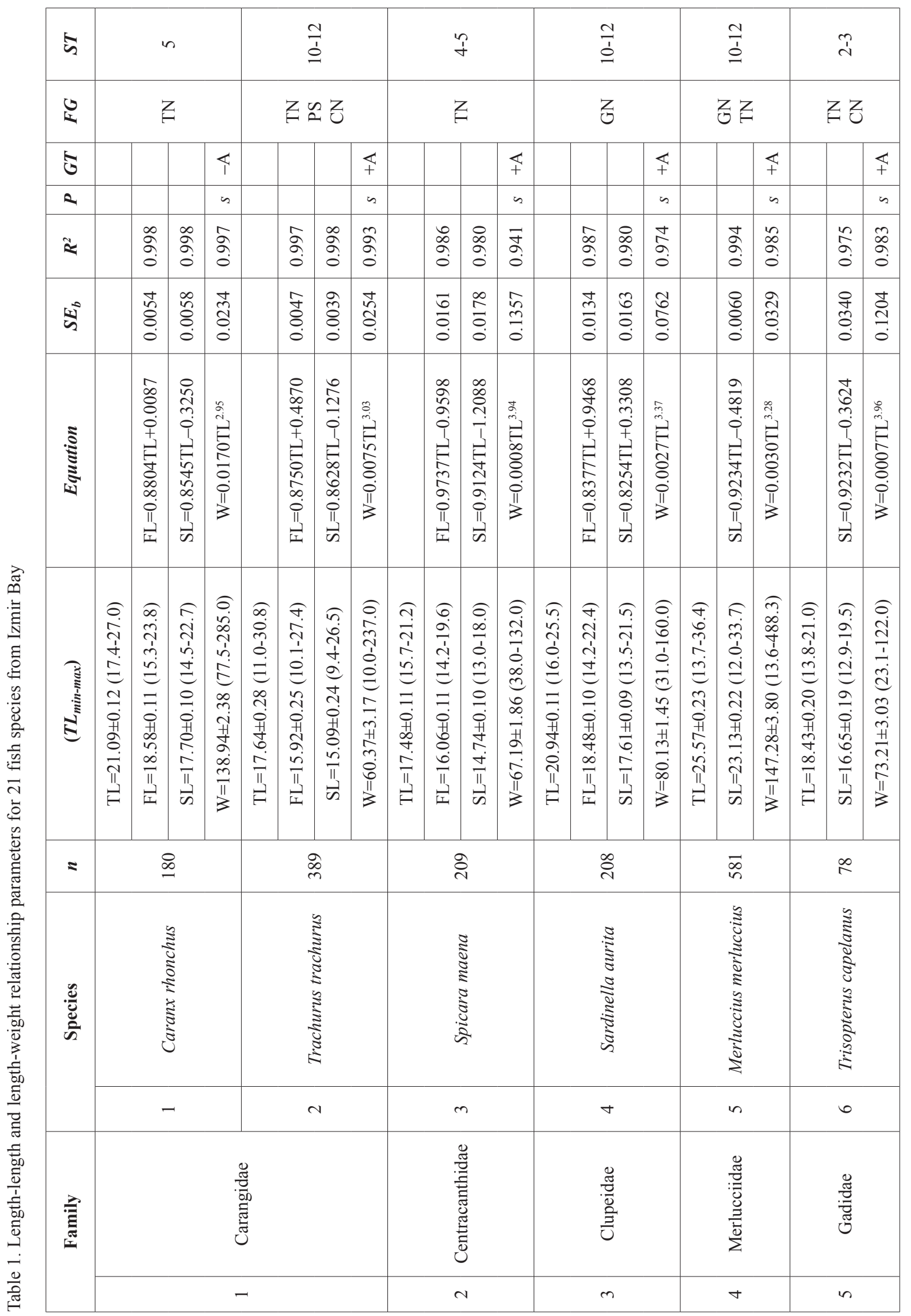




\begin{tabular}{|c|c|c|c|c|c|c|c|c|c|c|c|c|c|c|c|c|c|c|c|c|c|c|c|c|}
\hline $\bar{s}$ & \multicolumn{4}{|c|}{$=$} & \multicolumn{4}{|c|}{$\hat{b}$} & \multicolumn{4}{|c|}{$\frac{O}{\infty}$} & \multicolumn{4}{|c|}{$\frac{0}{a}$} & \multicolumn{4}{|c|}{$\frac{\mathfrak{I}}{\varrho}$} & \multicolumn{4}{|c|}{$\bar{\Xi}$} \\
\hline 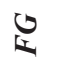 & \multicolumn{4}{|c|}{ Z } & \multicolumn{4}{|c|}{ Z } & \multicolumn{4}{|c|}{ Z } & \multicolumn{4}{|c|}{ 念Z } & \multicolumn{4}{|c|}{ 念Z } & \multicolumn{4}{|c|}{ Z } \\
\hline త & & & & $\neg$ & & & & $\neg$ & & & & $\varangle$ & & & & 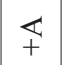 & & & & 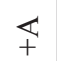 & & & & 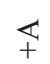 \\
\hline$a$ & & & & & & & & & & & & $n$ & & & & $n$ & & & & $n$ & & & & $n$ \\
\hline$\approx$ & & ڤ̆ & बे & ڤू̀े & & ڤू & ठ̀. & ڤ̆ & & ڤे & 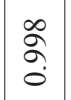 & $\begin{array}{l}n \\
2 \\
0 \\
0\end{array}$ & & $\vec{a}$ & $\begin{array}{l}\text { ț } \\
\text { ठे }\end{array}$ & ஃু & & $\stackrel{n}{\circ}$ & ڤ̆ & 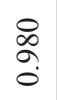 & & ڤे & ڤे & $\begin{array}{l}\infty \\
\stackrel{2}{\sigma}\end{array}$ \\
\hline है & & $\begin{array}{l}\overrightarrow{+} \\
\stackrel{8}{8} \\
\stackrel{0}{0}\end{array}$ & 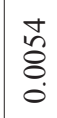 & 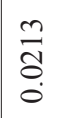 & & $\begin{array}{l}\stackrel{0}{0} \\
\stackrel{0}{0} \\
\stackrel{0}{0}\end{array}$ & $\begin{array}{l}0 \\
0 \\
0 \\
0 \\
0\end{array}$ & $\begin{array}{l}\infty \\
\stackrel{0}{+} \\
\stackrel{0}{0}\end{array}$ & & 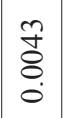 & $\begin{array}{l}\vec{n} \\
0 \\
0\end{array}$ & \begin{tabular}{l}
$n$ \\
\multirow{2}{f}{} \\
0 \\
0
\end{tabular} & & $\begin{array}{l}0 \\
\stackrel{0}{0} \\
0 \\
0\end{array}$ & $\begin{array}{l}\tilde{n} \\
\vdots \\
0\end{array}$ & 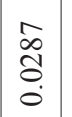 & & $\begin{array}{l}0 \\
8 \\
8 \\
0 \\
0\end{array}$ & $\begin{array}{l}\overrightarrow{0} \\
\stackrel{0}{0} \\
\stackrel{0}{0}\end{array}$ & 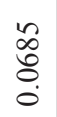 & & $\begin{array}{l}\mathcal{T} \\
\stackrel{0}{0} \\
\stackrel{0}{0}\end{array}$ & $\begin{array}{l}\overrightarrow{8} \\
8 \\
0\end{array}$ & $\begin{array}{l}\text { I } \\
\stackrel{0}{0} \\
\stackrel{0}{0}\end{array}$ \\
\hline 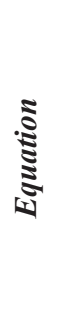 & & 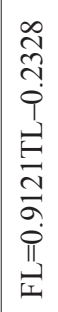 & 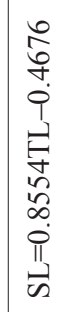 & 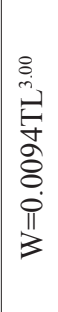 & & 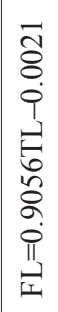 & 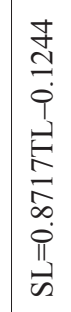 & 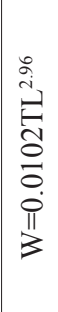 & & 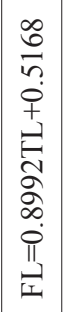 & 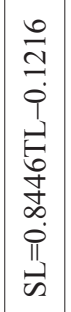 & 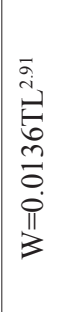 & & 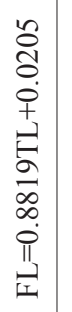 & 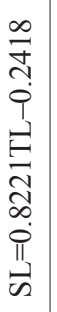 & 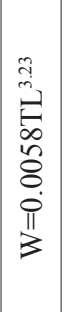 & & 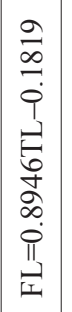 & 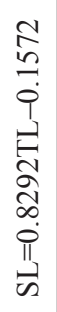 & 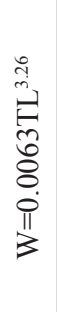 & & 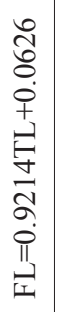 & 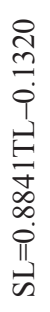 & 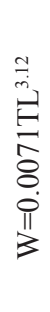 \\
\hline ڤ్ & 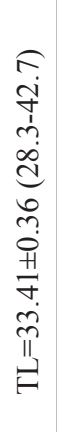 & 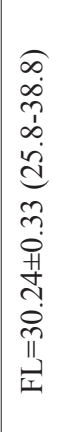 & 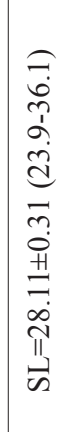 & 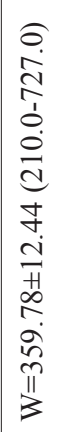 & 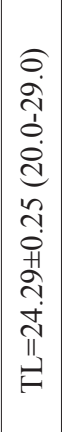 & 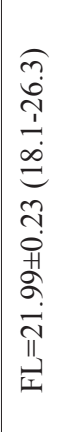 & 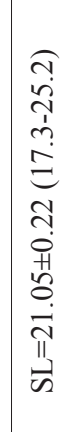 & 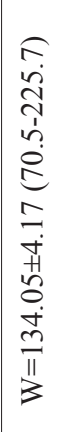 & 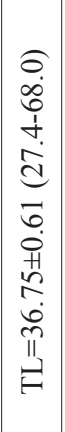 & 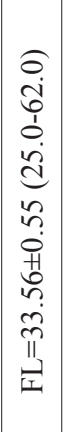 & 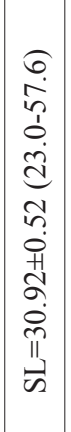 & 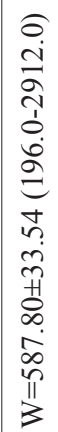 & 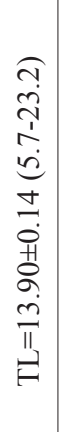 & 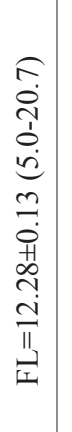 & 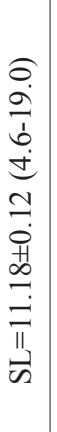 & 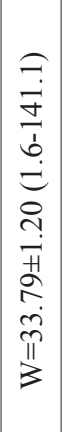 & 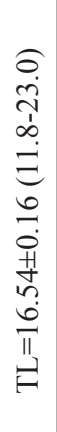 & 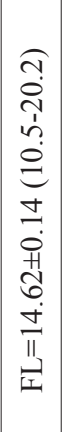 & 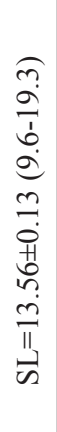 & 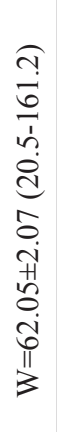 & 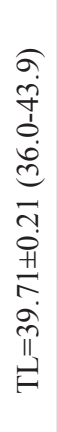 & 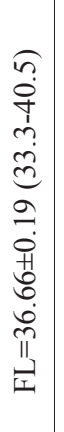 & 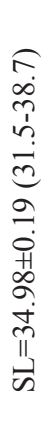 & 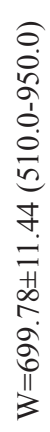 \\
\hline$=$ & \multicolumn{4}{|c|}{$\hat{\varrho}$} & \multicolumn{4}{|c|}{ 흐 } & \multicolumn{4}{|c|}{$\stackrel{\partial}{\vec{v}}$} & \multicolumn{4}{|c|}{$\stackrel{0}{\stackrel{9}{f}}$} & \multicolumn{4}{|c|}{$\infty$} & \multicolumn{4}{|c|}{$\hat{\varrho}$} \\
\hline \multirow[t]{2}{*}{ 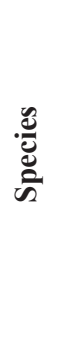 } & \multicolumn{4}{|c|}{ 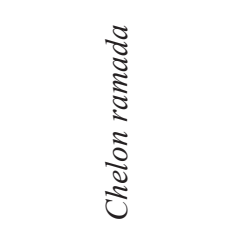 } & \multicolumn{4}{|c|}{$\begin{array}{l}\tilde{\Xi} \\
\tilde{\Xi} \\
\tilde{\Xi} \\
\tilde{\Xi} \\
\tilde{\Xi} \\
\tilde{\Xi}\end{array}$} & \multicolumn{4}{|c|}{ 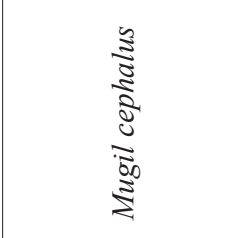 } & \multicolumn{4}{|c|}{ 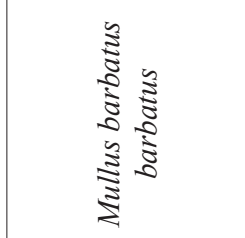 } & \multicolumn{4}{|c|}{ 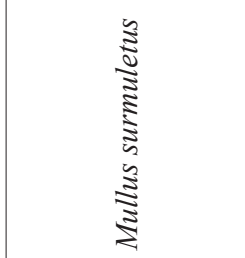 } & \multicolumn{4}{|c|}{ 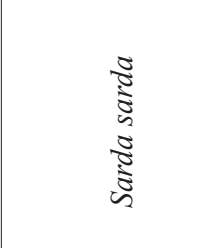 } \\
\hline & & r & $r$ & & & & $\infty$ & & & a & o & & & $\stackrel{0}{0}$ & & & & $=$ & & & & $\simeq$ & & \\
\hline \multirow[t]{2}{*}{ 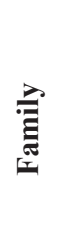 } & \multicolumn{12}{|c|}{ 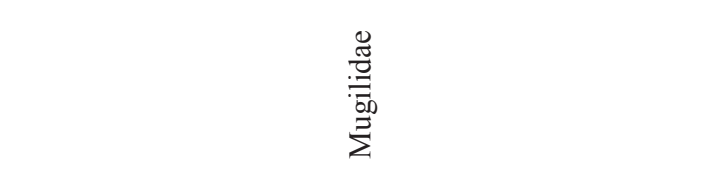 } & \multicolumn{8}{|c|}{ 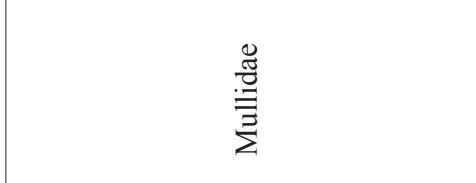 } & \multicolumn{4}{|c|}{ 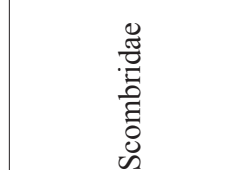 } \\
\hline & \multicolumn{12}{|c|}{0} & & & & $r$ & & & & & & $\infty$ & & \\
\hline
\end{tabular}




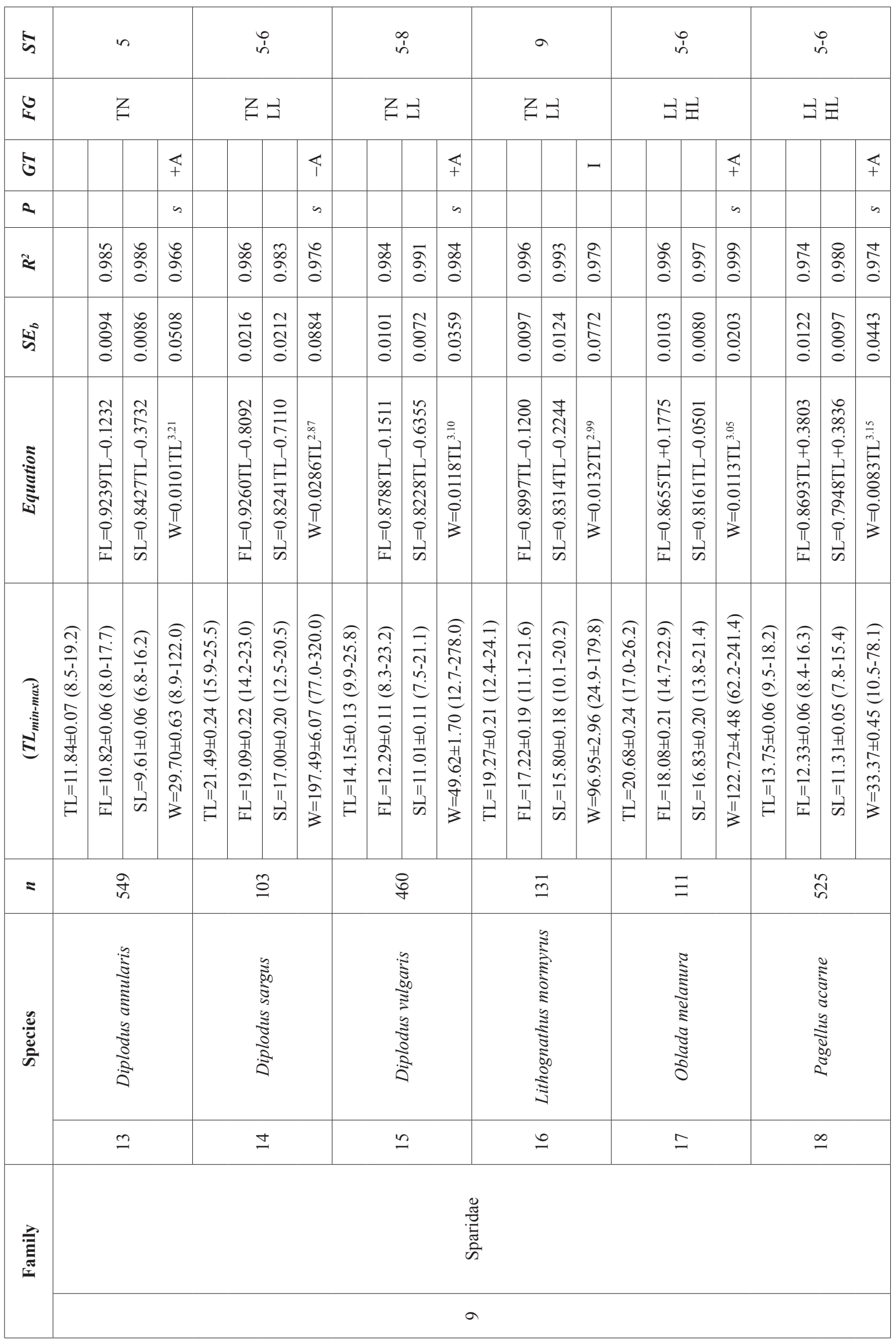




\begin{tabular}{|c|c|c|c|c|c|c|c|c|c|c|c|}
\hline 5 & \multicolumn{4}{|c|}{$\stackrel{?}{I}$} & \multicolumn{3}{|c|}{$\frac{7}{\varrho}$} & \multicolumn{4}{|c|}{$\frac{0}{a}$} \\
\hline ن & \multicolumn{4}{|c|}{ Zコ } & \multicolumn{3}{|c|}{ 互ヨ寻 } & \multicolumn{4}{|c|}{$\tilde{\Omega}$} \\
\hline ઉ & & & & 『 & & & $\underset{+}{\longleftarrow}$ & & & & « \\
\hline 2 & & & & $n$ & & & $n$ & & & & $n$ \\
\hline$\approx$ & & $\bar{\sigma}$ & 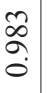 & 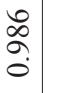 & & סे & $\begin{array}{l}0 \\
2 \\
0\end{array}$ & & \begin{tabular}{c}
$\hat{\infty}$ \\
\multirow{2}{0}{}
\end{tabular} & $\hat{\sigma}$ & 我 \\
\hline कै & & $\begin{array}{l}8 \\
\vdots \\
\vdots \\
\dot{0}\end{array}$ & $\begin{array}{l}0 \\
\infty \\
8 \\
0 \\
\dot{0}\end{array}$ & $\begin{array}{l}\tilde{a} \\
\tilde{\delta} \\
\dot{0}\end{array}$ & & 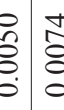 & $\begin{array}{l}\tilde{\delta} \\
\delta \\
0\end{array}$ & & $\begin{array}{c}\tilde{\infty} \\
\tilde{o} \\
0 \\
0\end{array}$ & 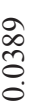 & $\frac{8}{d}$ \\
\hline 胥 & & 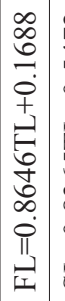 & 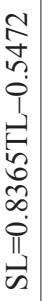 & 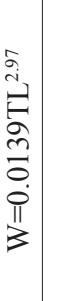 & $\begin{array}{l}0 \\
\vdots \\
0 \\
0\end{array}$ & 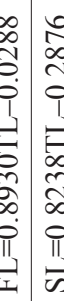 & $\frac{3}{3}$ & & 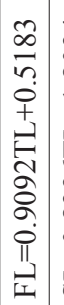 & 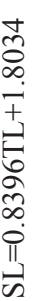 & 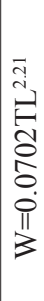 \\
\hline 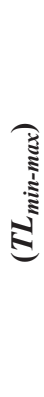 & 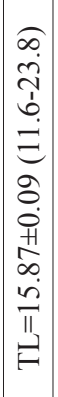 & 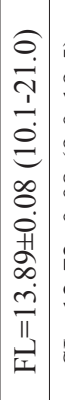 & 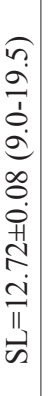 & 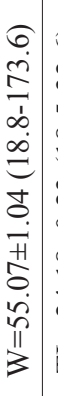 & 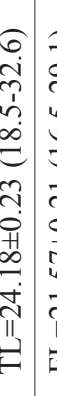 & 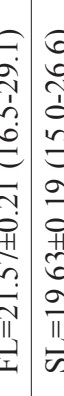 & 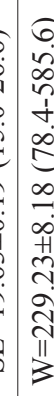 & 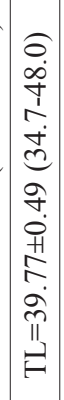 & 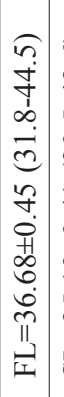 & 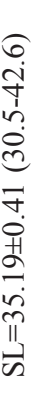 & 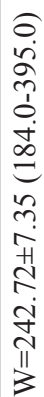 \\
\hline$=$ & & $\stackrel{ \pm}{\sim}$ & & & & $\overline{\widetilde{N}}$ & & & $i n$ & & \\
\hline 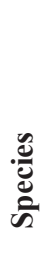 & & 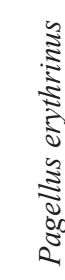 & & & & 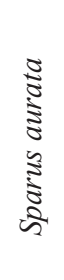 & & & 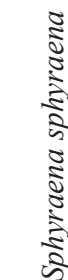 & & \\
\hline & & $\Omega$ & & & & ণ & & & $\bar{\sim}$ & & \\
\hline 䒿 & & & & $\begin{array}{l}\frac{\pi}{\pi} \\
\text { त्ञ } \\
\text { के }\end{array}$ & & & & & 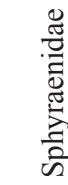 & & \\
\hline & & & & $a$ & & & & & 으 & & \\
\hline
\end{tabular}

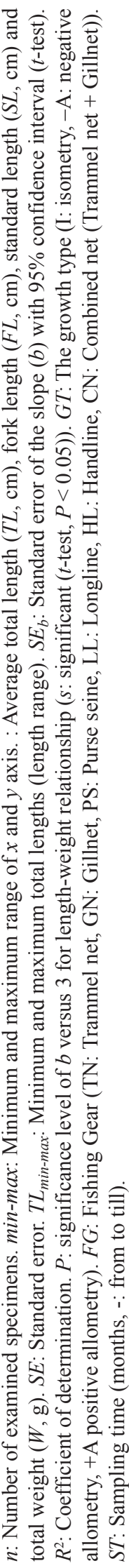

time, that could be explained by the fact that its body shape shows a negative allometric growth. In addition, the fact that data of $S$. sphyraena PETRAKIS \& STERGIOU (1995) was collected in the period from August to November which was longer than the present sampling period should be taken into account when the assessment is performed. As known from AVSAR (1998), body shape of any fish could determine its type of growth. Species with thinner and longer body form often show a negative allometric growth since its height grows greatly than its weight.

The high $b$ value in $S$. aurata was probably caused by the samples that having a narrow range of length. November and December, when the sampling was performed, were the periods in which sea bream was mostly fished in Izmir Bay, as they approached the shore in schools and migrated to spawning areas along the coastline. High condition factor could then influence an increase in the $b$ value. The $b$ values of most of the species presented in the present study are in general agreement with those observed from previous studies in Izmir bay and other adjacent areas.

The LWRs and LLRs for the 21 species are in FishBase (FROESE \& PAULY, 2016). However, there are only a few examples and one reference each for LL regressions of C. rhoncus and $S$. sarda in FishBase. The current study is believed to be likely to contribute greatly to increase in the number of references and samples.

Although some studies have been performed on the LWRs of fish in Izmir Bay, there are only two on LLRs. These are KARA \& BAYHAN (2008) for Boops boops and BAYHAN \& KARA (2015) for Sarpa salpa. The current study will be important especially for length-length relationships of 21 fish species in Table 1 . There is only one length-weight relationship found for 
the species Trisopterus capelanus in FishBase. However, there are no length-length relationships for it. This study will thus provide lengthlength relationships for the first time on the species T. capelanus. Although FishBase provides relationships between SL and FL measurements for the species Sarda sarda, there are no values for correlation between measurements of TL and FL and those between TL and SL length. Likewise, FishBase presents relationships between TL and FL for Sparus aurata, but there are no values for the relationship between TL and SL for the species. Moreover, FishBase has only one fish length-length relationships value for Chelon saliens and Caranx rhonchus. This study provided increasing the number of length-length relationship values for the above-mentioned species.

In conclusion, the study provides us with a basic knowledge of LW and LL relationships involving 21 species, which are greatly likely of use for fisheries biologists and managers to study in Izmir Bay.

\section{ACKNOWLEDGMENTS}

We do appreciate their precious contributions to the processes of sampling and measurement by Tilki Yaşar and Ganga Ibrahim from Şemikler Fishing Port, Celal Güven from Urla Port, Deve Ünal, the Chief of Güzelbahçe Fisheries Cooperative and his wife, Yurdanur Görgün, the Sales Manager in the same cooperative.

\section{REFERENCES}

AVSAR, D. 1998. Fisheries Biology and Population Dynamics (in Turkish), University of Cukurova, Faculty of Fisheries, Adana, Turkey, $303 \mathrm{pp}$.

BAGENAL, T. \& F. W. TESCH. 1978. Age and growth. In: Bagenal T. (Editor). Method for Assessment of Fish Production in Freshwater. IBP Handbook, Blackwell Scientific Press, Oxford, pp. 101-136.

BAYHAN, B. \& A. KARA. 2015. Length-weight and length-length relationships of the salema Sarpa salpa (Linnaeus, 1758) in Izmir Bay (Aegean Sea of Turkey). Pak. J. Zool., 47: 1141-1146.

BEYER, J.E. 1987. On length-weight relationships. Part I: Computing the mean weights of the fish in a given length class. Fishbyte, 5: 11-13.

BINOHLAN, C. \& D. PAULY. 1998. The length-weight table. In: R. Froese and D. Pauly (Editors). FishBase 1998: Concepts, Design and Data Sources. ICLARM. Manila, pp. 121-123.

FROESE, R. 1998. Length-weight relationships for 18 less-studied fish species. J. Appl. Ichthyol., 14: 117-118.

FROESE, R. 2006. Cube law, condition factor and weight-length relationships: History, metaanalysis and recommendations. J. Appl. Ichthyol., 22: 241-253.
FROESE, R., A.C. TSIKLIRAS \& K.I. STERGIOU. 2011. Editorial note on weight-length relations of fishes. Acta Ichthyol. Piscat., 41: 261-263.

FROESE, R. \& D. PAULY. 2016. FishBase. World Wide Web electronic publication. Version 06/2016. [cited 2016 Jun 22]. Available from: http://www.fishbase.org.

HILE, R. 1936. Age and growth of the cisco, Leucichthys artedi (Le sueur), in the lakes of the Northeastern Highlands, Wisconsin. Bull. US Bur. of Fish., 48: 211-317.

KARA, A. \& B. BAYHAN. 2008. Length-weight and length-length relationships of the bogue Boops boops (Linnaeus, 1758) in Izmir Bay (Aegean Sea of Turkey). Belg. J. Zool., 138: 154-157.

KEIVANY, Y., M. AALIPOUR, M. SIAMI \& S.S. MORTAZAVI. 2015a. Length-weight relationships for three species from Beheshtabad River, Karun River Drainage, Iran. Iran. J. Ichthyol., 2: 296-298.

KEIVANY, Y., A. NEZAMOLESLAMI, S. DORAFSHAN \& S. EAGDERI. 2015b. Length-weight and length-length relationships in populations of Garra rufa from different rivers and basins of Iran. Int. J. Aquat. Biol., 3: 409-413.

KOUTRAKIS, E.T. \& A.C. TSIKLIRAS. 2003. Lengthweight relationships of fishes from three northern Aegean estuarine systems (Greece). J. Appl. Ichthyol., 19: 258-260. 
MARTIN, W. R. 1949. The mechanics of environmental control of body form in fishes. Univ. Toronto Stud. Biol., 58: 1-91.

MOUTOPOULOS, D.K. \& K.I. STERGIOU. 2002. Length-weight and length-length relationships of fish species from the Aegean Sea (Greece). J. Appl. Ichthyol., 18: 200-203.

OZAYDIN, O. \& E. TASKAVAK. 2006. Lengthweight relationships for 47 fish species from Izmir Bay (eastern Aegean Sea, Turkey). Acta Adriat., 47: 211-216.

PAZI, I., G. YUCEL-GIER \& Y. ARISOY. 2010. The use of marine in the Izmir Bay. In: L. Balas (Editor). Turkey's Marine and Coastal Area VIII. National Congress, Trabzon, Turkey, 27 April-1 May, Kay Türkiye Milli Komitesi Press, İstanbul, Turkey, pp. 317-322.

PETRAKIS, G. \& K.I. STERGIOU. 1995. Weightlength relationships for 33 fish species in Greek Waters. Fish. Res., 21: 465-469.

RICKER, W. E. 1979. Growth rates and models. In: W.S. Hoar, D.J. Randall and J.R. Brett (Editors). Fish Physiology, Vol. VIII: Bioenergetics and Growth. Academic Press. London, pp. 677-743.

SARKAR, U.K., R.S. NEGI, P.K. DEEPAK, W.S. LAKRA \& S.K. PAUL. 2008. Biological parameters of the endangered fish Chitala chitala (Osteoglossiformes: Notopteridae) from some Indian rivers. Fish. Res., 90: 170-177.

STERGIOU, K.I. \& D.K. MOUTOPOULOS. 2001. A review of length-weight relationships of fishes from Greek marine waters. Naga ICLARM Q., 24: 23-39.

TESCH, F.W. 1971. Age and growth. In: W.E. Ricker (Editor). Methods for Assessment of Fish Production in Fresh Waters. Blackwell Scientific Publications, Oxford, pp. 99-130.

WANG, T., H.-S. WANG, G.-W. SUN, D. HUANG \& J.-H. SHEN. 2012. Length-weight and length-length relationships for some Yangtze River fishes in Tian-e-zhou Oxbow, China. J. Appl. Ichthyol., 28: 660-662.

YUCEL-GIER, G., Y. ARISOY \& I. PAZI. 2010. A spatial analysis of fish farming in the context of ICZM in the Bay of Izmir-Turkey. Coast. Manage., 38: 399-411.

\title{
Dužinsko-maseni i dužinsko-dužinski odnos za 21 vrstu riba ulovljenih u Izmirskom zaljevu
}

\author{
Ali KARA*, Deniz ACARLI , Akın Türker İLKYAZ i Ali Özcan BABAOĞLU \\ *Kontakt,e-pošta: ali.kara@ege.edu.tr
}

\section{SAŽETAK}

U ovom radu se prikazuje povezanost dužinsko-masenog odnosa i dužinsko-dužinskog odnosa za 21 vrstu riba ulovljenih u Izmirskom zaljevu. Koeficijenti a i b za odnos dužinsko-masenog (LWR) i odnos dužinsko-dužinskog (LLR) izračunati su s formulama $\mathrm{W}=\mathrm{a} / \mathrm{b}$ i kao jednadžbom $\mathrm{TL}=\mathrm{a}+$ $\mathrm{bFL}$ i $\mathrm{TL}=\mathrm{a}+\mathrm{bSL}$. Jednadžbe za pretvaranja standardne duljine i vilične duljine $\mathrm{u}$ ukupnu duljinu i obrnuto, pokazale su se linearnima. Uključeni odnosi bili su značajno međusobno povezani (R2> 0,942). Vrijednosti b u LWR varirale su od 2,21 do 3,96 (srednja vrijednost \pm SE: 3,15 $\pm 0,08$ ). Studentov t-test pokazao je da se $87,9 \%$ vrijednosti $b$ značajno razlikuje od 3 .

Ključne riječi: riba, dužinsko-dužinski odnos, dužinsko-maseni odnosi, Izmirski zaljev 